\title{
Karl F. Stock, Rudolf Heilinger, Marylène Stock: Personal- bibliographien österreichischer Frauen in Kultur, Wissenschaft und Politik. Graz: Verlag der Technischen Universität Graz, 2020. ISBN: 978-3-85125-760-1
}

In den Personalbibliographien österreichischer Persönlichkeiten von Stock, Heilinger, Stock, an denen von den zwei Bearbeitern und der Bearbeiterin seit 1962 gearbeitet wird, sind zum Zeitpunkt der Fertigstellung des vorliegenden Werkes 18.758 Persönlichkeiten mit rund 78.000 Personalbibliographien nachgewiesen. Davon sind 1.781 Frauen aus den Bereichen Kunst und Kultur, Wissenschaft und Politik mit 7.076 Bibliographien und Werkverzeichnissen zu finden. Das Werk beginnt mit einer interessanten Einbegleitung von Bernhard Reismann, dem Archivar der Technischen Universität Graz.

Der Verlag der Technischen Universität Graz veröffentlicht anlässlich des Gedenkens an „Hundert Jahre Frauenwahlrecht und Frauenstudium“ ein Werk gemeinsam mit einer Ausstellung im Frühjahr 2020. Damit der wertvolle Beitrag von Frauen in Kultur, Wissenschaft und Politik in Österreich verdeutlicht wird, entstand diese Begleitpublikation der „Personalbibliographien österreichischer Frauen in Kultur, Wissenschaft und Politik“. Dichter brachten seit früher Zeit ihr Frauenlob zum Ausdruck, doch die Wertschätzung der Frauen erreichte noch lange nicht das Niveau der Männerwelt, daran änderte das Frauenwahlrecht und das Frauenstudium nicht viel. Beispiele finden wir in der österreichischen Bundeshymne ...,,Heimat bist du großer Söhne" oder in der Ode an die Freude von Schiller/Beethoven ..., ,alle Menschen werden Brüder“. In beiden Beispielen blieben Frauen unerwähnt. Die Wertschätzung der Frauen muss in Gedanken, Worten und Werken erfolgen. KF Stock fasste diese Bemühungen schon 2015 in folgende Worte:

Wer kennt die Töchter, nennt die Frauen auf die wir Österreicher schauen wir danken vieles unsern Schönen nicht weniger als unsern Söhnen 
Nach dem alphabetischen Verzeichnis der Autorinnen (S. 477) folgen 5 Register: Geburtsdaten, Geburtsorte, Todesjahre, Todesorte und das Allgemeine Gesamtregister, S. 527-907. Dazu noch eine Besonderheit: das Werk zeigt im Frontcover einen Farblinolschnitt der Venus $(11,4$ x 7,4 cm), im Text, S. 477 und am Backcover einen Schwarz-weiß-Linolschnitt, sowie auf S. 907 am Schluss aller Register einen kleinen kreisrunden Linolschnitt, alle aus der künstlerischen Werkstatt von KF Stock.

Mit der vorliegenden Personalbibliographie leistet das AutorInnenteam einen wichtigen Beitrag zur Betonung der Wertschätzung der österreichischen Frauen in Kultur, Wissenschaft und Politik. Im Werk zu blättern und zu lesen ist ein Vergnügen.

Dr. ${ }^{\text {in }}$ Sigrid Reinitzer E-Mail: sigrid.reinitzer@gmail.com

DOI: https://doi.org/10.31263/voebm.v73i2.4296

(c) Sigrid Reinitzer

Dieses Werk ist - exkl. einzelner Logos und Abbildungen - lizenziert unter einer Creative-Commons-Lizenz Namensnennung 4.0 International 